\title{
Influence of Deep Cryogenic Treatment on Microstructures and Mechanical Properties of an Ultrafine-Grained WC-12Co Cemented Carbide
}

\author{
Hejia Zhang $\cdot$ Liqing Chen $\cdot$ Jing Sun $\cdot$ Wenguang Wang $\cdot$ Quanzhao Wang
}

Received: 27 June 2014/Revised: 3 August 2014/Published online: 16 September 2014

(C) The Chinese Society for Metals and Springer-Verlag Berlin Heidelberg 2014

\begin{abstract}
Effect of deep cryogenic treatment (DCT) on the microstructures and mechanical behavior of ultrafine-grained WC-12Co cemented carbide was investigated by using XRD, SEM, and DSC. The phase transformations of pure Co and binder phase Co in cemented carbide were analyzed in detail to correlate the strengthening mechanism with its $\alpha \rightarrow \varepsilon$ phase transition. The results show that DCT resulted in a slight increase in hardness and bending strength of ultrafinegrained WC-12Co cemented carbide. For the ultrafine-grained cemented carbide after DCT, there is no significant change in the microstructure and the elemental distribution of the cemented carbides, but the fractured morphology shows a feature of plastic deformation. In the cases of pure Co and the binder phase Co in WC-12Co cemented carbide, they exhibit different features of phase transformation. The improvement of mechanical property of cemented carbide can be attributed to the increased amount of $\varepsilon$-Co in WC-12Co composites after DCT.
\end{abstract}

KEY WORDS: WC-12Co composite; Cemented carbide; Deep cryogenic treatment; Mechanical properties; Microstructure

\section{Introduction}

Cemented carbide is a kind of composite material prepared by powder metallurgy route, which usually contains refractory metallic carbide particles, e.g., high-hardness WC as reinforcement and high-ductility Co as binder phase $[1,2]$. The cemented carbide has excellent mechanical and

Available online at http://link.springer.com/journal/40195

H. Zhang $\cdot$ L. Chen $(\bowtie) \cdot$ J. Sun

State Key Laboratory of Rolling and Automation, Northeastern University, Shenyang 110819, China

e-mail: lqchen@mail.neu.edu.cn

W. Wang

School of Mechanical Engineering, Liaoning Shihua University, Fushun 113001, China

Q. Wang

Institute of Metal Research, Chinese Academy of Sciences,

Shenyang 110016, China physical properties, e.g., high hardness and strength, high resistance to corrosion at elevated temperatures, and low coefficient of thermal expansion, and so they have wide applications in many industrial fields [3]. The cemented carbide has been used as the main cutting tool material in high-speed machining tools, among which the ultrafinegrained cemented carbide tools are considered as the highend products due to their more superior wear resistance and higher hardness and strength than conventional cemented carbides.

Although the ultrafine-grained cemented carbide used as cutting tool material possesses excellent mechanical property and wear-resistance, wear is still the main failure mechanism in the process of metal cutting when using ultrafine-grained cemented carbide tools, especially in highspeed refractory metal cutting applications. High-speed cutting behavior can be improved by gradient design of the cutting tool or coating of carbonitride on the tool surface. However, the complicated preparation will undoubtedly lead to increase in the manufacturing cost and limit its large-scale 
application. Therefore, further improvement on the wear resistance of ultrafine-grained cemented carbide tools remains as a challenge to be resolved in high-speed cutting tool production and its application.

Deep cryogenic treatment (DCT) is a simple and viable process, by which the wear resistance, hardness, strength, and dimensional stability can be improved. DCT is usually conducted at extreme temperature lower than $-183{ }^{\circ} \mathrm{C}$ by treating the materials according to the predesigned processing schedule [4-7]. DCT has been successfully used for alloying steels including tool and bearing steels [8-10], and the results showed that the service life of these steels were substantially extended [11].

As for the other materials, e.g., cemented carbide tools, although some studies indicated that the wear resistance of cemented carbide can be obviously improved by DCT [12-14], there are still some differences regarding the service life while using DCT. This may be related to the chemical composition, DCT process, and working condition of the cemented carbide tools. For example, in two independent studies, Yong et al. [4, 6] noted that the DCT of tungsten carbide inserts improves tool life (flank wear) performance, and resistance to chipping in milling and turning to a certain extent. They stated that tools under mild cutting conditions stood to gain from DCT, but heavy-duty cutting operations with long periods of heating of the cutting tool would not benefit from it.

As for the influence of DCT on ultrafine-grained cemented carbide, there are very few reports about it. From the limited research results available, one may find that there exists a great difference in the strengthening mechanisms. As reported in Ref. [5], DCT can prevent the removal of the cobalt binder when cutting, but the reason remains unknown. Thakur et al. [14] believed that this could be due to cobalt densification or shrinkage that holds the carbide particles more firmly and thereby increases the wear resistance properties of the insert. Some other studies indicated that the changes in mechanical behavior of cemented carbide are closely related to the transformation of Co binder phase and the increase of the surface residual stresses after DCT $[13,15]$.

In most existing studies, it is believed that the strengthening is mainly related to phase transformation of Co $[13,15,16]$. In fact, there has been lack of strong evidence, and the relation of Co phase transformation with temperature could not be observed. In XRD phase analysis, due to the existence of WC particles, the diffraction peaks of Co are almost fully overlapped with WC with the result that the phase transformation of Co could not be determined. As a result, further researches are required to understand the effect of DCT on ultrafine-grained cemented carbide and the related mechanism.

In this study, the effect of DCT on the microstructure and mechanical properties of ultrafine-grained $\mathrm{WC}-12 \mathrm{Co}$ cemented carbide was first investigated. A detailed study was made on the phase transformation of binder phase Co over a wide temperature range using thermal analysis and selective electrolytic corrosion technology. The strengthening mechanism of cemented carbide processed by DCT was finally analyzed.

\section{Experimental}

The raw materials used in this study are pure cobalt plate and ultrafine-grained WC-12Co cemented carbide. The ultrafine-grained WC-12Co cemented carbide was produced by low-pressure sintering in Xiamen Golden Egret Special Alloy Co., Ltd., and its grain size was $0.4 \mu \mathrm{m}$. The mass proportions of $\mathrm{WC}$ and $\mathrm{Co}$ are 87 and $12 \mathrm{wt} \%$, respectively. The inhibitors for grain growth have a total fraction of $1 \mathrm{wt} \%$. The cemented carbide was first directly put into liquid nitrogen at ambient temperature and held for different times. Afterward, it was taken out and then exposed to air. The hardness of WC-12Co cemented carbide was measured before and after DCT in KB3000BVRZ-SA universal hardness meter. The bending strength was tested in Instron 1343 servo hydraulic universal testing machine (sample size is $4.5 \mathrm{~mm} \times 4.5 \mathrm{~mm}$ $\times 40 \mathrm{~mm}$, and the loading speed is $1 \mathrm{~mm} / \mathrm{min}$ ).

Microstructural observation and elemental analysis were performed in ZEISS ULTRA-55 F20 field emission scanning electron microscope (SEM) and JEOL JXA-8530F electron probe (EP), respectively. The volume changes of pure Co transformed in heating and cooling processes were examined using Formastor-FII automatic transformation testing instrument. At first, pure Co plate was heated up to $1,100{ }^{\circ} \mathrm{C}$ at $10{ }^{\circ} \mathrm{C} / \mathrm{s}$ and held for $5 \mathrm{~min}$ and then cooled to room temperature at $2.5^{\circ} \mathrm{C} / \mathrm{s}$. SETARAM simultaneous thermal analyzer testing system was used to analyze heat flow changes of WC$12 \mathrm{Co}$ and pure $\mathrm{Co}$ during heating and cooling. Both the specimens were heated up to $1,100{ }^{\circ} \mathrm{C}$ at $5^{\circ} \mathrm{C} / \mathrm{min}$ and held for $15 \mathrm{~min}$ and then cooled down to room temperature at the rates of $5{ }^{\circ} \mathrm{C} / \mathrm{min}$ and $10{ }^{\circ} \mathrm{C} / \mathrm{min}$, respectively. In the $\mathrm{X}$-ray phase analysis, Electromet 4 electrolytic polishing etching machine was used to conduct selective electrolytic corrosion for WC-12Co cemented carbide in order to avoid the effect of overlapped diffraction peaks of binder phase Co and WC phase.

\section{Results and Discussion}

\subsection{Mechanical Behaviors Before and After Deep Cryogenic Treatment}

Figure 1 shows the change in Vickers hardness value of ultrafine-grained WC-12Co cemented carbide with time for 


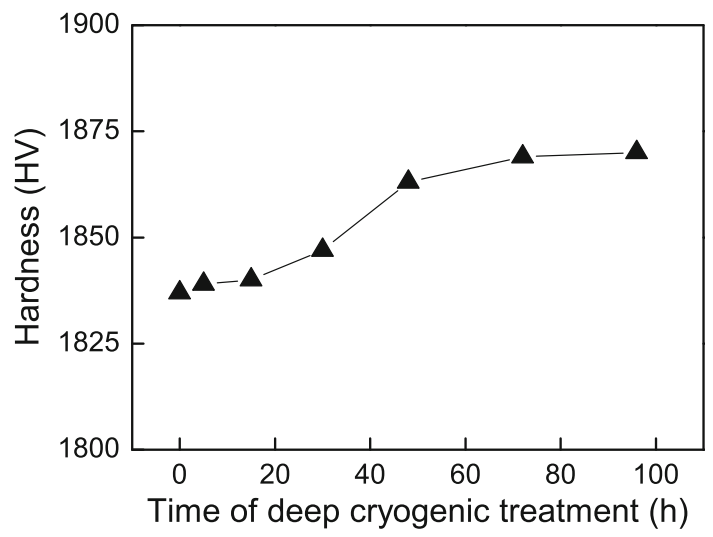

Fig. 1 The hardness change of the ultrafine-grained WC-12Co cemented carbide with time for deep cryogenic treatment

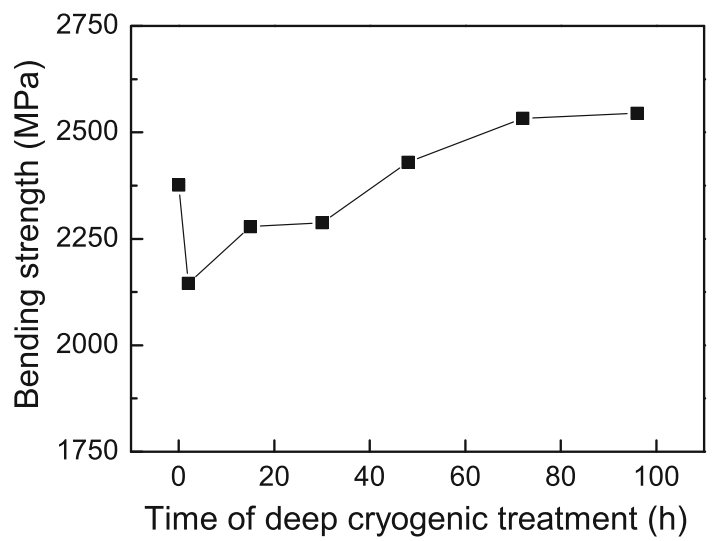

Fig. 2 Relationship between bending strength and deep cryogenic treatment time for ultrafine-grained WC-12Co cemented carbides

DCT. After DCT, there is a slight increase in the Vickers hardness value of cemented carbide. The Vickers hardness value is $1,837 \mathrm{HV}$ before DCT, while it is $1,867 \mathrm{HV}$ after $72 \mathrm{~h}$ of DCT. The Vickers hardness is hardly increased with any further extension of the DCT time.

Variation of the bending strength with time for DCT is displayed in Fig. 2 for the ultrafine-grained WC-12Co cemented carbide. It can be seen from Fig. 2 that the bending strength generally increases with the DCT time. However, there is a slight decrease at the initial stage of DCT. When the time for DCT is $2 \mathrm{~h}$, the bending strength is $2,145 \mathrm{MPa}$. After $72 \mathrm{~h}$ of DCT, the bending strength almost reaches the maximum of $2,532 \mathrm{MPa}$, which is higher than 2,376 MPa prior to DCT. The variation of the bending strength with the DCT time for ultrafine-grained cemented carbide obtained in this study is similar to that reported in Ref. [16].

The above experimental result indicates that DCT does have a certain influence on the mechanical property of ultrafine-grained cemented carbide. This effect can be reflected by microstructural change or phase transformation within the cemented carbide. It is known that Co phase in cemented carbide has two kinds of structures, i.e., $\alpha$-Co phase with fcc structure and $\varepsilon$-Co phase with hcp structure [15]. Slip systems in $\varepsilon$-Co is less than that in $\alpha$-Co and is hard to deform, which is characterized by its high hardness and strength. No phase transformation or structural change occurs in brittle and hard WC particles over a wide temperature range. Almost the entire cemented carbide consists of WC particles and binder phase Co, with the latter possessing good toughness as well as plasticity. Therefore, it is reasonable to deduce that the change of mechanical properties is largely due to the structural transformation of the Co phase.

In the earlier stage of DCT, a sudden drop in the bending strength of cemented carbide may be caused by the damage from thermal shock impact, when the cemented carbide is promptly quenched into liquid nitrogen [17]. The subsequent increase of the bending strength with the DCT time possibly results from $\varepsilon$-Co phase transformation. In the earlier stage of DCT, the $\varepsilon$-Co transformation is not so much, and the mechanical performance is thus mainly determined by thermal shock damage. With extension of the DCT time, the amount of $\varepsilon$-Co is increased which leads to the further improvement of the mechanical properties.

For the cemented carbide processed by DCT, the alien atoms dissolved in Co will precipitate owing to decreased solubility, which reduces lattice distortion and microcracks in $\varepsilon$-Co phase. Thus, the plasticity of $\varepsilon$-Co and strength of cemented carbides are improved. Moreover, the precipitated alien atoms can play a role in strengthening like second-phase particles and further enhance the strength and wear resistance by increasing the hard phase in Co binder phase and hindering dislocation motion. However, it is very difficult to observe such tiny particles precipitated during DCT.

\subsection{Micro-morphology and Distribution of Alloy Elements}

In order to explore the microstructural changes of ultrafinegrained WC-12Co cemented carbide after DCT, the fractured surfaces were observed and alloying elemental distribution analyzed in cemented carbide by using SEM and EP.

Figure 3 shows the morphologies of the fractured surfaces of cemented carbide before and after DCT. It can be seen from the Fig. 3 that there is no obvious change in terms of the WC particle size before and after DCT. Compared with features before DCT, however, plenty of plastically torn ligaments are found on the fractured surfaces of WC particles after DCT, as indicated by arrows in 

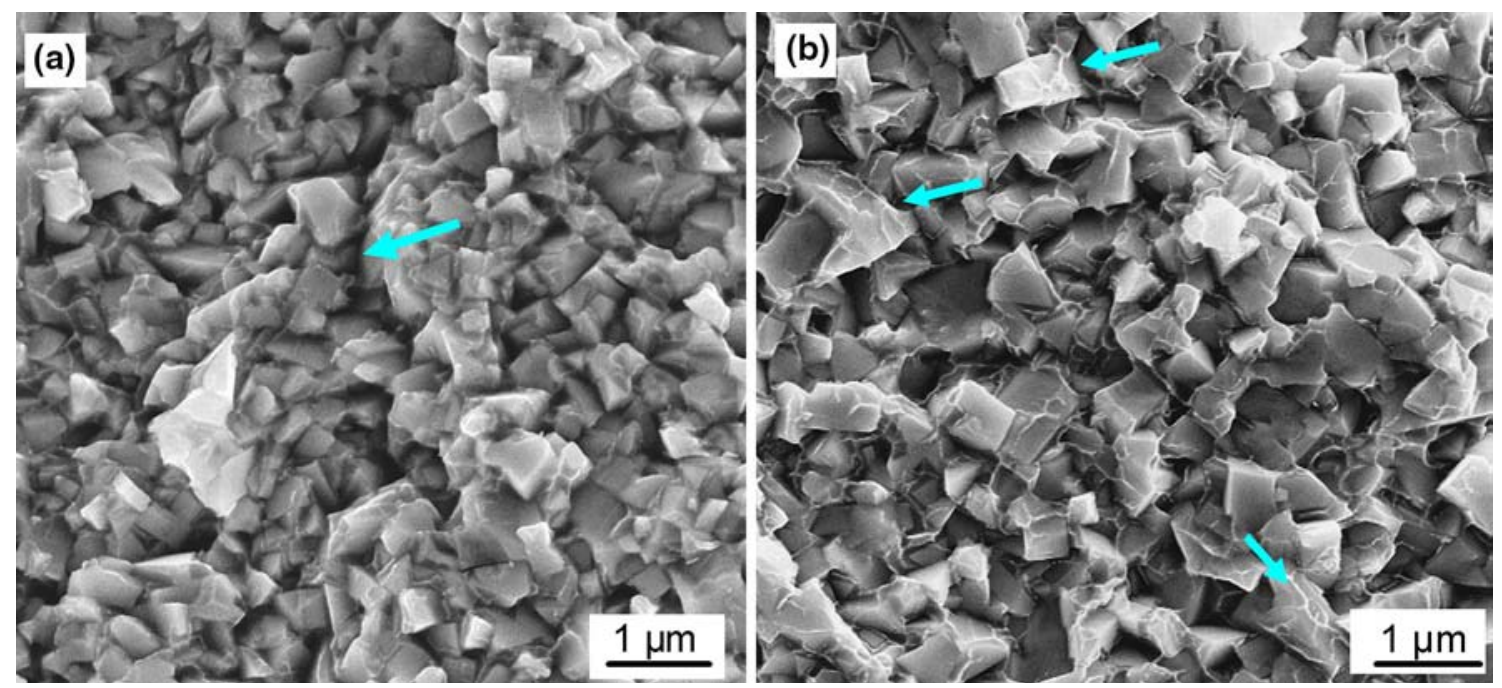

Fig. 3 SEM micrographs showing the fracture surfaces of WC-12Co cemented carbide: a as-sintered; b deep cryogenically treated in liquid nitrogen for $72 \mathrm{~h}$
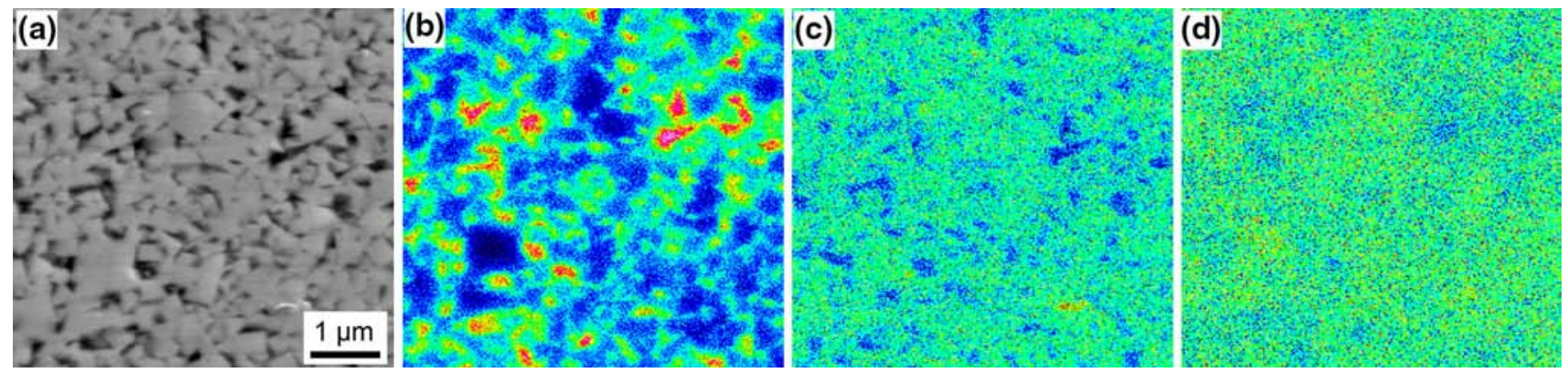

Fig. 4 SEM micrograph and elemental distributions of cemented carbides before deep cryogenic treatment: a microstructure; $\mathbf{b}$ Co distribution; c C distribution; $\mathbf{d} \mathrm{W}$ distribution

Fig. 3b. For the fractured surfaces of WC particles before DCT, as shown in Fig. 3a, there seem to be not many ligaments, and there are brittle cracks on the fractured surfaces. The retained plastic ligaments are Co binder phase, rather than WC particles, since WC particles are of hard and brittle phase and have obviously no tearing morphology. As for the binder phase $\mathrm{Co}$, it has ability to plastically deform to some extent. Owing to the good wettability of Co with WC, these two phases can be firmly combined when the cemented carbide suffers fracture. It is clear that the ductility and toughness of cemented carbides were higher after DCT. This may be owing to the crystal structure resetting of Co after phase transformation and elimination of dislocations and other defects. In addition, the strength of $\varepsilon$-Co phase is also higher than that of $\alpha$-Co phase.

Figures 4 and 5 show the surface morphologies and distributions of alloying elements of WC-12Co cemented carbides before and after DCT, respectively. The surface morphology before DCT is shown in Fig. 4a, while the elemental distributions of $\mathrm{Co}, \mathrm{C}$, and $\mathrm{W}$ corresponding to Fig. $4 \mathrm{a}$ are, respectively, shown in Fig. $4 \mathrm{~b}$, c, and d.
Figure 5 shows the morphology and elemental distributions after DCT. As shown in Figs. 4a and 5a, the dark gray WC phase displays angular shape and the black Co binder phase was surrounded by the WC phase. It can be seen that the WC particle size, morphology, and distribution of alloying elements do not show any change before and after DCT. For the cemented carbide, whether DCT is conducted or not, there is hardly any Co element in WC particles. However, the Co binder phase may contain very few $\mathrm{W}$ and $\mathrm{C}$ elements. Due to the limitations in magnification, the precipitated tiny second-phase particles could not be observed in Co binder phase. Some studies have indicated that a change in WC particle size and occurrence of $\eta$ phase in the matrix after DCT were observed $[18,19]$. However, they could not be confirmed in this experiment.

\subsection{Phase Transformation of Co and DSC Analysis of Cemented Carbide}

Figure 6 shows the variation of temperature vs. volume of Co measured by the full automatic quenching dilatometer. 

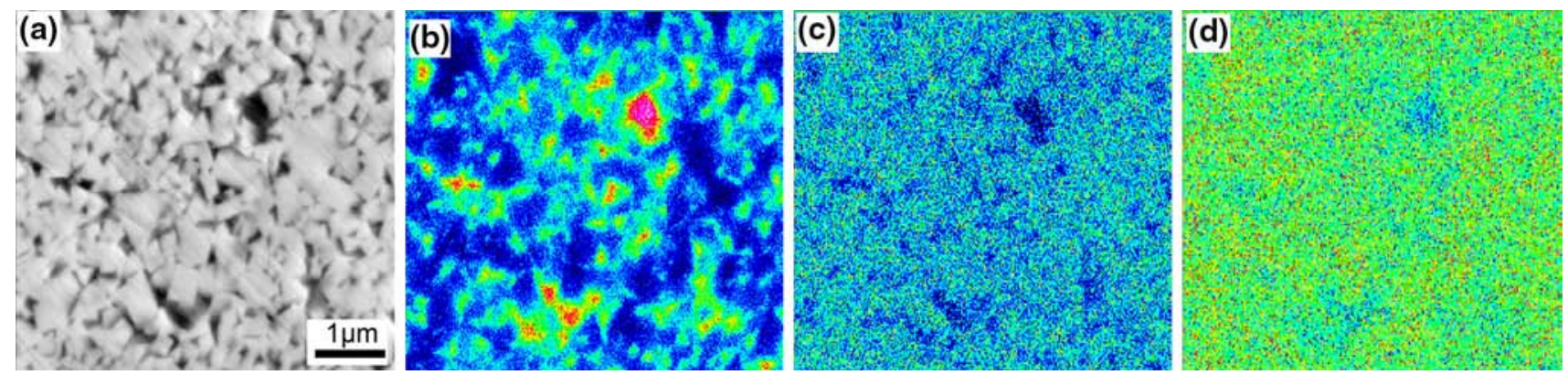

Fig. 5 SEM micrograph and elemental distribution of cemented carbides after deep cryogenic treatment for 72 h: a microstructure; b Co distribution; c C distribution; $\mathbf{d} \mathrm{W}$ distribution

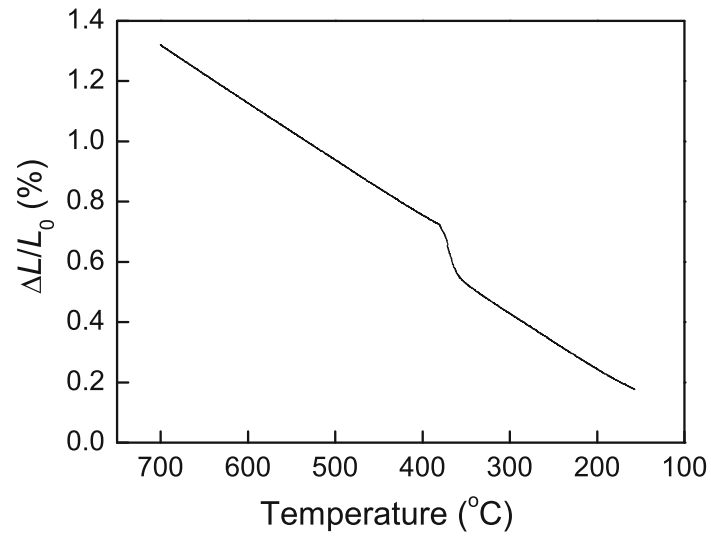

Fig. 6 Phase transition curve of Co in cooling process

During the experiment, Co was first heated to $1,100{ }^{\circ} \mathrm{C}$ and held for $10 \mathrm{~min}$. Then it was cooled down to room temperature at $2.5^{\circ} \mathrm{C} / \mathrm{s}$. It can be seen from this curve that the volume of Co was changed when it was cooled down to $\sim 400{ }^{\circ} \mathrm{C}$. This indicates that there was a phase transformation, and this phase transformation was completed in a narrow temperature range, i.e., high-temperature stable phase $\alpha$-Co transformed to low-temperature stable phase $\varepsilon$ Co.

DSC curves exhibiting the temperature-heat flux curves of cemented carbides and pure Co are presented in Fig. 7 for the cooling process. The DSC result of pure Co cooled at the rate of $10{ }^{\circ} \mathrm{C} / \mathrm{min}$ is depicted as curve (1) in Fig. 7, while those of cemented carbide cooled at the rates of 10 and $5{ }^{\circ} \mathrm{C} / \mathrm{min}$ are, respectively, drawn as curves (2) and curve (3). From Fig. 7, it can be seen that a phase transformation occurred in pure $\mathrm{Co}$ at $\sim 400{ }^{\circ} \mathrm{C}$, and this phase transformation was completed in a very narrow temperature range as characterized by heat flux. This result is consistent with that measured above by fully automatic quenching dilatometer. Furthermore, an obvious change in heat flux curves can be observed at $\sim 800{ }^{\circ} \mathrm{C}$ for the cemented carbides, when it was cooled either at

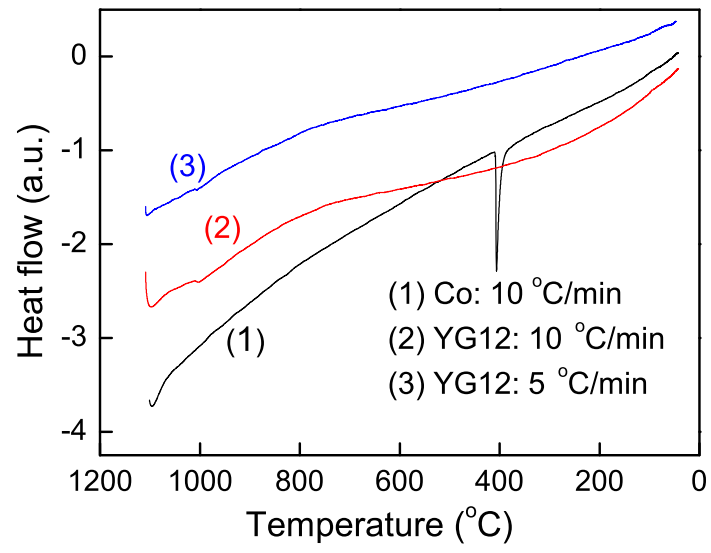

Fig. 7 Heat flow curves of cemented carbide and pure Co during cooling

the rate of 10 or $5{ }^{\circ} \mathrm{C} / \mathrm{min}$. This indicates that there exists phase transformation in Co during this time, and a continual change of heat flux is maintained till room temperature.

As the cooling rate in curve (2) is higher than that in curve (3), the transformed amount in curve (2) is much larger than that in curve (3) in the same time. In terms of the slope, the slope of curve (2) is also greater than that of curve (3). As the phase transformation in pure $\mathrm{Co}$ is completed in a very short time and has a larger amount, it shows a sharp change in slope of heat flux curve. After completion of phase transformation, the curve becomes straight. In contrast, the heat flux curves of cemented carbide still do not become straight even when room temperature is reached. Thus, it is easy to know that the phase transformation of cemented carbide is still not fully completed at room temperature, which is mainly attributed to the pinning effect of WC particles on the grain boundaries of Co phase. So a further decrease in temperature is required for the subsequent transformation of residual $\alpha$ Co $\rightarrow \varepsilon$-Co. 


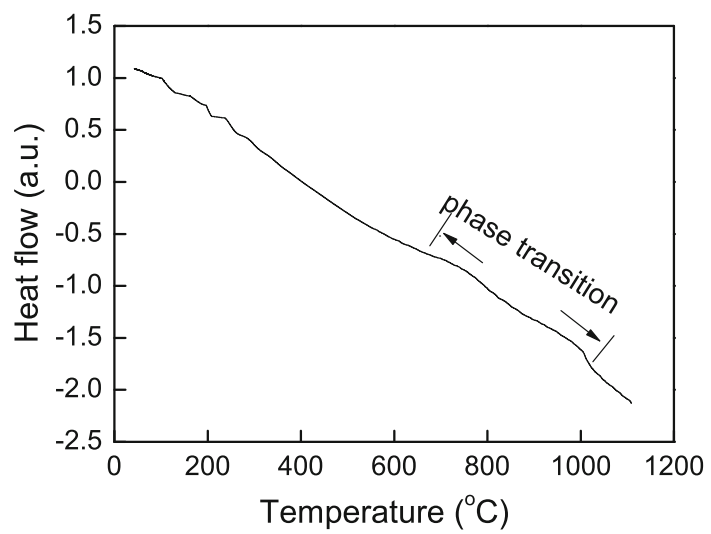

Fig. 8 Heat flow curve of cemented carbide in reheating process

A DSC curve showing change of heat flux with temperature in reheating process (heating rate is $5{ }^{\circ} \mathrm{C} / \mathrm{min}$ ) for ultrafine-grained WC-12Co cemented carbide is depicted in Fig. 8. It indicates that a fluctuating heat flux curve occurred in the earlier stage of heating. Afterward, the heat flux curve remains stable with the increasing temperature. When the temperature is close to $400{ }^{\circ} \mathrm{C}$, the heat flux curve remains in a stable state, which shows that no phase transformation takes place at this time. When the temperature reaches about $700{ }^{\circ} \mathrm{C}$, the heat flux curve begins to raise upward, which shows that a phase transformation has happened in cemented carbide at this time. When the temperature is raised to $1,000{ }^{\circ} \mathrm{C}$ or so, the curve becomes stable again, which indicates that phase transformation is completed at this time. Compared with phase transformation in pure $\mathrm{Co}$, there is a great difference in phase transformation of binder phase $\mathrm{Co}$ in cemented carbide. Therefore, in the cutting process, reverse transformation from $\varepsilon$-Co to $\alpha$-Co will not occur until the tool temperature of cemented carbide exceeds $700{ }^{\circ} \mathrm{C}$, and thus, it will not have any influence on DCT of the tools.

\subsection{Phase Analysis Before and After Deep Cryogenic Treatment}

Owing to diffraction overlapping of $\mathrm{WC}$ and Co, it is very difficult to observe the diffraction peak of the binder phase Co in XRD analysis of cemented carbide. Therefore, there is a need to use selective electrolytic corrosion to treat the cemented carbide. In this way, plenty of WC particles can be corroded and thus the diffraction peak of Co can be observed. Figure 9a shows the XRD spectrum of the cemented carbide without DCT, which indicates that the crystal structures of Co are totally bec $\alpha$-Co, and the diffraction peaks of $\varepsilon$-Co can hardly be observed. In contrast, Fig. 9b shows the XRD spectrum of the cemented carbide after $72 \mathrm{~h}$ of DCT. According to the phase analysis, there are no other phases

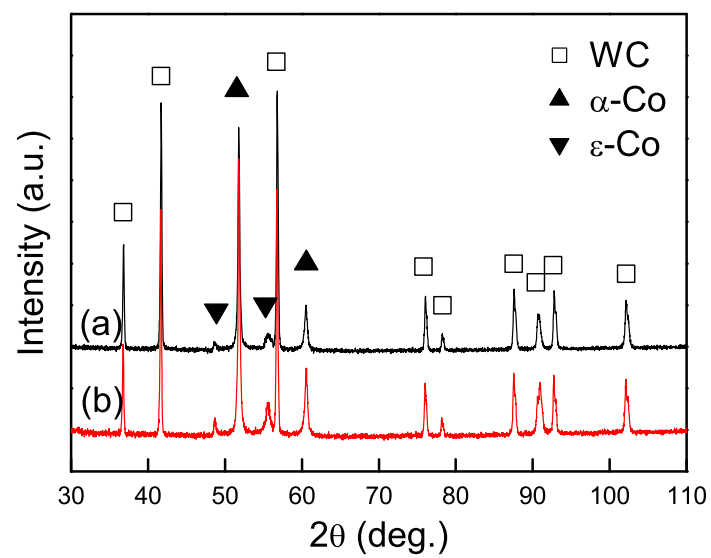

Fig. 9 XRD spectra of WC-12Co cemented carbides before and after cryogenic treatment for $72 \mathrm{~h}$

except for binder phase Co and WC particles after DCT. Compared with the untreated state, however, diffraction peaks of $\varepsilon$-Co obviously appeared, although there was almost no change in the diffraction peaks of WC before and after DCT. Based on this, it can be concluded that DCT can really promote transformation of $\varepsilon$-Co, and there is no influence on the crystal structure of WC particles.

In such a case, we may note that mechanical property of cemented carbides can be improved by DCT due to the increased amount of $\varepsilon$-Co phase. In other words, the change of the mechanical property depends mainly on phase transformation of binder phase Co. The most important point is that $\varepsilon$-Co has lower friction coefficient, good lubricity, and is hard to wear, and it can improve the wear resistance of the materials greatly. At temperature higher than $417{ }^{\circ} \mathrm{C}$, the $\alpha$-phase in Co has lower free energy, and therefore Co exists in the form of $\alpha$ phase. At temperature below $417^{\circ} \mathrm{C}$, the high-temperature stable $\alpha$-phase transforms to $\varepsilon$-phase with lower free energy. The binder phase Co in cemented carbides has a strong resistance to phase transformation caused by pinning effect of ultrafinegrained WC particles and alien atoms solid solved in $\alpha$ phase. So large amount of $\alpha$-phase exists in binder phase Co at room temperature, since the $\alpha \rightarrow \varepsilon$ phase transformation of cemented carbide is restrained in the cooling process after sintering. DCT can further increase the difference in Gibbs free energy values between $\alpha$-phase and $\varepsilon$ phase, thereby providing the driving force for phase transformation, in other words, the transformed amount of $\varepsilon$ phase is increased.

\section{Conclusions}

(1) After DCT, there is a slight increase both in hardness and bending strength for ultrafine-grained WC-12Co 
cemented carbide. At the beginning of the DCT, there is a sudden drop in bending strength caused by the damage from thermal shock. The bending strength is hardly increased after $72 \mathrm{~h}$ of DCT.

(2) For the ultrafine-grained WC-12Co cemented carbide after DCT, there is no significant change in the microstructure and elemental distribution, but the fractured morphology shows a feature of plastic deformation.

(3) The pure Co and the binder phase Co in WC-12Co cemented carbide exhibit different features of phase transformation. The phase transformation of $\alpha \rightarrow \varepsilon$ in pure Co can be completed in a very narrow temperature range, while the change of the binder phase Co in WC-12Co cemented carbide strongly depends on temperature, and a wide temperature range is required for the Co phase change. The improvement in the mechanical property of cemented carbide can be attributed to the increased amount of $\varepsilon$-Co phase in WC-12Co composites after DCT.

Acknowledgments This work was financially supported by the Major Special Project, Ministry of Industry and Information Technology, China (No. 2012ZX04003061).

\section{References}

[1] R. Bao, J.H. Yi, Y.D. Peng, H.Z. Zhang, A.K. Li, Trans. Nonferrous Met. Soc. China 22, 853 (2012)
[2] R. Bao, J.H. Yi, Y.D. Peng, H.Z. Zhang, Trans. Nonferrous Met. Soc. China 23, 372 (2013)

[3] S.X. Zhao, X.Y. Song, J.X. Zhang, X.M. Liu, Acta Metall. Sin. 43, 107 (2007). (in Chinese)

[4] A.Y.L. Yong, K.H.W. Seah, M. Rahman, Int. J. Adv. Manuf. Technol. 32, 638 (2007)

[5] J. Gisip, R. Gazo, H.A. Stewart, J. Mater. Process. Technol. 209, 5117 (2009)

[6] A.Y.L. Yong, K.H.W. Seah, M. Rahman, Int. J. Mach. Tools Manuf 46, 2051 (2006)

[7] S.S. Gill, R. Singh, H. Singh, J. Singh, Int. J. Mach. Tools Manuf. 49, 256 (2009)

[8] D.N. Collins, Heat Treat. Met. 23(2), 40 (1995)

[9] N.S. Kalsia, R. Sehgalb, V.S. Sharma, Mater. Manuf. Process. 25, 1077 (2010)

[10] H.A. Stewart, Forest Prod. J. 54, 53 (2004)

[11] S.S. Gill, H. Singh, R. Singh, J. Singh, Int. J. Adv. Manuf. Technol. 48, 175 (2010)

[12] T.V.S. Reddy, T. Sornakumar, M.V. Reddy, R. Venkatram, A. Senthilkumar, Mach. Sci. Technol. 13, 269 (2009)

[13] J.S. Liu, Y.X. Pu, M.F. Tan, Hot Working Technol. 41(6), 184 (2012). (in Chinese)

[14] D. Thakur, B. Ramamoorthy, L. Vijayaraghavan, Mater. Lett. 62, 4403 (2008)

[15] Y. Jiang, D. Chen, Mater. Sci. Eng. A 528, 1735 (2011)

[16] Z.H. Chen, L. Fan, Y. Jiang, L.M. Wang, W. Peng, X.H. Zhou, Cem. Carbide 27(01), 1 (2010). (in Chinese)

[17] J.W. Wei, L.L. Tang, S.H. Li, X.C. Wu, Trans. Nonferrous Met. Soc. China 22, 2421 (2012)

[18] T.V.S. Reddy, T. Sornakumar, M.V. Reddy, R. Venkatram, Int. J. Refract. Met. Hard Mater. 27(1), 46 (2009)

[19] T.V.S. Reddy, T. Sornakumar, M.V. Reddy, R. Venkatram, Cryogenics 48(9-10), 458 (2008) 